\title{
Um Modelo de Dinâmica de Sistemas para Previsão de Desgaste em Pavimentos Rodoviários no Brasil.
}

\section{A System Dynamics Model to Predict Brazilian Highway Conditions}

Márcio Augusto Lima Teodoro1, Rafael Pinheiro Amantéa², Gisele Tessari Santos $^{3}$, Sérgio Luiz Araújo Vieira ${ }^{4}$, Rodrigo Diógenes Rezende ${ }^{5}$

\section{RESUMO}

Este trabalho tem como objetivo utilizar a Modelagem Dinâmica de Sistemas como ferramenta de previsão de desgaste em pavimentos rodoviários no Brasil. Um Modelo de Dinâmica de Sistemas desenvolvido para o sistema de gestão rodoviária nos Estados Unidos foi adaptado à política de gestão rodoviária Brasileira. A modelagem proposta tem com objetivo contribuir para uma alocação cuidadosa dos recursos financeiros, bem como melhorar as atuais metodologias brasileiras utilizadas na engenharia rodoviária. Para determinar e simular as condições do pavimento considerou-se, no modelo, parâmetros como idade do pavimento, taxas de manutenção, taxa de deterioração e relações causais entre as variáveis de tráfego. Foram utilizados dados experimentais para calibrar os parâmetros do modelo e consequentemente validar o modelo proposto. Os resultados da modelagem proposta permitiram apontar momentos de possíveis ocorrências de taxas críticas de deterioração na rodovia em estudo, facilitando, assim, decisões de manutenção estratégica e operacional. Este trabalho, portanto, tem uma importância substancial para o planejamento técnico, político e financeiro da gestão de pavimentos rodoviários no Brasil, proporcionando uma alternativa aos métodos tradicionais utilizados na área.

Palavras-chave: Dinâmica de Sistemas. Pavimentos. Simulação.

\section{ABSTRACT}

This work aims to apply the Systems Dynamic Modeling as a tool for determining Brazilian road conditions. A System Dynamics Model created for the road management system in the United States was adapted to the Brazilian benchmark's road management politics. The model should contribute to careful funding allocation, as well as to present an improvement in the current Brazilian methodologies used by road engineering. To determine and simulate the pavement conditions, parameters such as pavement age, maintenance rates, deterioration rate and causal relations of traffic variables were considered by the proposed model. Experimental data were used to calibrate the model parameters. The results of the proposed modeling allowed to point out moments of potential occurrences of critical deterioration rates, facilitating strategical and operational maintenance decisions. This work has substantial support to technical, political and financial planning, providing an alternative to the traditional methods of managing road pavements in Brazil.

Keywords: System Dynamics. Simulation. Pavement.
${ }^{1}$ Mestre - Instituto de Educação Tecnologica - IETEC.

${ }^{2}$ Doutor - Instituto de Educação Tecnológica - IETEC.

rafaelamantea@yahoo.com.br

${ }^{3}$ Doutor - Instituto de Educação Tecnológica - IETEC.

${ }^{4}$ Doutor - Instituto Brasileiro de Mercados e Capitais - IBMEC

${ }^{5}$ Mestre - Instituto de Educação Tecnologica - IETEC. 


\section{INTRODUÇAOO}

No Brasil, o transporte rodoviário é predominante diante dos outros modais de transporte visto que, na segunda metade do século $X X$, houve um grande investimento público em implantação de rodovias para interligar as várias regiões do país. Ainda hoje, pode-se destacar que a porção mais representativa de suas instalações de transporte concentra-se no modal rodoviário com contribuição aproximada de $60 \%$ do total de toneladas transportadas por quilômetro (IBGE, 2014).

Uma vez que o solo natural, onde se situam as rodovias, não é suficientemente resistente para suportar a repetição de cargas de roda sem sofrer deformações, a sua pavimentação garante a trafegabilidade em qualquer condição climática, e proporciona aos usuários das vias conforto e segurança. A construção desta estrutura, denominada pavimento, é feita sobre o subleito para suportar as cargas dos veículos de forma a distribuir essas cargas nas diversas camadas do pavimento (CRONEY, 1977).

A malha rodoviária brasileira foi estimada como sendo da ordem de 1,7 milhões de quilômetros de rodovias dos quais apenas $214.000 \mathrm{~km}$ (12\% do total) são pavimentados. Considerando a parcela não pavimentada (1,4 milhões de quilômetros), tem-se $93 \%$ de rodovias municipais 3 estaduais e 7\% federais (CNT, 2018).

Apesar de o Brasil ter a quarta maior malha rodoviária do mundo, nota-se uma ausência significativa de novas ferramentas e inovação nos processos de gerenciamento de rodovias no país. Considerando a importância em melhorar continuamente os sistemas de conservação, manutenção e restauração de rodovias no Brasil, faz-se necessária a utilização de técnicas de modelagem e predição na gestão de pavimentos.

Nas últimas cinco décadas, a quase totalidade dos estudos sobre o comportamento dos pavimentos asfálticos tiveram como base estudos empíricos e testes de fadiga em laboratório, também conhecidos como métodos "empírico-mecanísticos" (SALINI, 2010). estudo moderno dos pavimentos foi iniciado por volta de 1958, com a construção da pista experimental da American Association of State Highway Officials (AASHO), posteriormente denominada "AASHTO" (CAREY; IRICK, 1960; CAREY; IRICK, 1962). Tais estudos, eram adequados à realidade da época que era caracterizada por limitações tecnológicas, baixo custo dos materiais e mão-de-obra, e pequeno tráfego nas estradas, entretanto, não são mais compatíveis com os desafios do século XXI.

Outro fator que vem sendo estudado há muito tempo no processo de degradação de pavimentos é a influência dos fatores ambientais, uma vez que os pavimentos rígidos e 
flexíveis alteram seus estados de tensões e deformações quando expostos a gradientes térmicos (MALLICK et al. 2014). Para tanto, torna-se indispensável o estudo das condições climáticas e ambientais relacionadas aos materiais e estruturas.

Segundo Bastos, Soares e Barroso (2014), foram desenvolvidos estudos para um novo método brasileiro mecanístico-empírico de dimensionamento de pavimentos, com lançamento no ano de 2016. Apesar disso, ainda não existe no Brasil um consenso sobre os modelos de desempenho mais apropriados.

De acordo Sterman (2000), os estudos físicos das dinâmicas de deterioração do pavimento foram feitos em conjunto com as operações de manutenção de nível macro, e essas combinações permitiram a construção de modelos de simulação fundamentados na física das operações de estradas que podem ser validadas considerando os fatores sociais e gerenciais (FALLAH-FINI; TRIANTIS, 2009).

Devido à limitação de recursos para a conservação, operação e gestão das estradas e infraestruturas, exige-se o uso de várias ferramentas das áreas de engenharia, gestão e princípios de economia para ajustar os orçamentos dos estados e municípios (LEE et al, 2012).

Os novos desafios do século XXI, com a crescente quantidade de veículos nas estradas, aumento da capacidade de carga dos caminhões, altos custos dos materiais utilizados nos pavimentos, elevados custos da mão-de-obra e as crescentes restrições ambientais à extração e uso de jazidas de materiais, impõem a necessidade de melhoria na qualidade das predições da vida de serviço e modelagem dos pavimentos asfálticos (MADEH PIRYONESI e EL-DIRABY, 2017). Isso não significa que os atuais métodos empírico-mecanísticos para pavimentos sejam totalmente inúteis. Eles são úteis desde que consideradas as limitações dos mesmos (SALINI et al., 2010).

Devido ao comportamento ultra complexo dos pavimentos asfálticos, não é possível obter a qualidade desejada nas predições, utilizando somente abordagens empíricas ou empírico-mecanísticas. Para isso, são necessárias ferramentas robustas capazes de manusear todas as variáveis relevantes e suas praticamente infinitas combinações (YANG et al., 2003; BOSURGI; TRIFIRÒ, 2005). Técnicas avançadas de inteligência evolutiva, têm sido utilizadas na área de pavimentação e para melhoria estratégica de construção e manutenção de estradas (LI et al 2019).

$\mathrm{O}$ uso destes tipos de abordagens pode proporcionar melhorias significativas para os tomadores de decisões, que vão desde a fase de planejamento de uma implantação, até 
as etapas de conservação e manutenção, racionalizando a aplicação de recursos e identificando pontos chaves do processo.

De acordo com Lopes et al. (2015), apesar dos avanços tecnológicos e da ampliação das redes viárias, as decisões com relação à manutenção e reabilitação de pavimentos ainda têm sido tomadas apenas com base na experiência de alguns profissionais e, principalmente, por razões políticas. No quadro atual, a gerência é feita de forma aleatória, com o sentido de resolver as necessidades mais extremas de reparo, com pouco planejamento prévio.

Segundo Friedman (2003), a modelagem de gestão de pavimentos é uma atividade essencial para um sistema de gestão de pavimentos. Por meio da modelagem, pode-se planejar tanto a manutenção de rotina quanto a reabilitação integral das estradas. Os modelos de gestão de pavimentos permitem o desenvolvimento de planos de longo prazo e a estimativa dos resultados de processos de gestão de pavimento. Além disso, esses modelos ajudam na tomada de decisões em relação a fatores responsáveis pela degradação dos pavimentos em estudo.

Segundo Fallah-Fini et al. (2010), a manutenção eficaz das estradas depende de diversas atividades, incluindo a compreensão do estado atual do pavimento e a previsão de suas condições futuras, além das decisões para melhoria na forma de alocação de recursos limitados para operações de manutenção.

A relevância deste trabalho se deve ao fato de o Brasil ter a $4^{\mathrm{a}}$ maior malha rodoviária do mundo com sua infraestrutura altamente deteriorada, com grandes restrições orçamentárias e um tráfego intenso de veículos levando a uma necessidade emergente de melhoria no desempenho das práticas de manutenção de rodovias (Fallah-Fini et al. 2015). Para atender a esta demanda, modelos de dinâmica de sistemas que buscam compreender a dinâmica do desgaste do pavimento, compreender a forma que se relacionam os fatores que aceleram ou reduzem o desgaste do pavimento e que buscam até mesmo propor políticas ótimas de manutenção e alocação de recursos para a manutenção foram desenvolvidos(FRIEDMAN, 2003; FALLAH et al., 2010; FALLAH, 2015).

Dessa maneira, a utilização de modelos de dinâmica de sistemas aliados às técnicas tradicionais pode apresentar uma alternativa para a melhoria na gestão de pavimentos no Brasil, sendo uma boa recomendação o uso de um sistema híbrido nos contratos de manutenções rodoviárias.

O presente trabalho tem como objetivo, portanto, desenvolver um modelo de dinâmica de sistemas para auxiliar a tomada de decisão na área de gestão de pavimentos no Brasil, 
contribuindo, assim, para o desenvolvimento e aprimoramento de aplicação de recursos, e para melhoria das atuais ferramentas utilizadas pelos profissionais da engenharia rodoviária no Brasil.

De maneira específica, este trabalho se compromete a utilizar dados de um trecho rodoviário do centro-oeste de Minas Gerais (MG 818 entre os municípios de Florestal e Pará de Minas-MG) para o desenvolvimento e validação do modelo proposto.

\section{MATERIAIS E METODOS}

\subsection{A MODELAGEM POR MEIO DE DINÂMICA DE SISTEMAS}

O processo de modelagem de um problema por meio da Dinâmica de Sistemas se inicia pela escolha das variáveis que estão envolvidas de maneira direta ou indireta com o problema em questão. Esta seleção tem por objetivo criar um "diagrama causal" ou um "diagrama de causa e efeito" de maneira que possa se conhecer as possíveis relações entre as variáveis e também os "loops" ou laços de realimentação do sistema.

As relações causais em um diagrama de causa e efeito podem ser facilmente compreendidas. Uma relação causal positiva entre uma variável A e outra variável B simboliza que um aumento no valor da variável $A$ implica em um aumento no valor da variável B. A relação também é caracterizada como positiva caso ocorra uma diminuição em $A$ e consequente diminuição em B. Observa-se na figura 1 a representação de uma relação causal positiva.

A relação é caracterizada como negativa quando um aumento na variável $A$ acarreta em uma diminuição na variável $B$. A diminuição em $A$ e consequente aumento em $B$ também caracteriza uma relação causal negativa.

Figura 1 - Exemplo de relação causal positiva

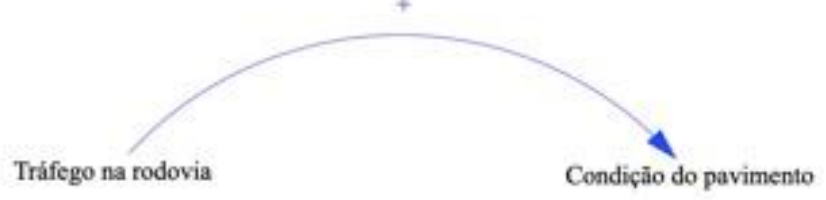

Fonte: Elaborado pelo autor.

Na medida em que o diagrama é construído, podem ocorrer os chamados "loops" ou "malhas de retroalimentação". Estes elementos surgem das relações causais entre as variáveis e se comportam como reforço ou controle para alguma decisão. As malhas de 
retroalimentação podem ser positivas ou negativas. A classificação das mesmas envolve a contagem do número de relações causais negativas. Dizemos que um "loop" é positivo quando o número de relações causais que o compõe é par. O "loop" é considerado negativo quando o número de relações causais que o compõe é ímpar. As malhas ou loops positivos mostram que o comportamento do sistema apresentará crescimento ou diminuição, de forma exponencial. Já as malhas ou "loops" negativos mostram um comportamento estabilizador, controlando os comportamentos exponenciais e levando o sistema a um patamar de equilíbrio.

Os diagramas causais são utilizados para representar as hipóteses das causas dinâmicas, modelos mentais e os comportamentos do sistema em estudo (STERMAN, 2000).

Figura 2 - Exemplo de ciclos de retroalimentação

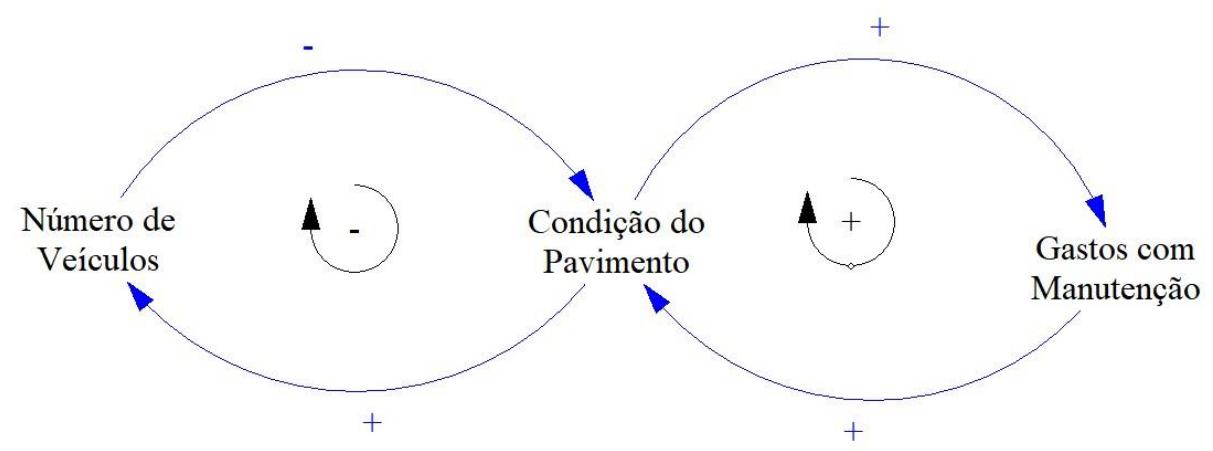

Fonte: Elaborado pelo autor

\subsection{O MODELO CAUSAL PARA A PREDIÇÃO DO DESGASTE DO PAVIMENTO}

A condição do pavimento foi modelada levando-se em consideração as relações causais dentre as variáveis associadas aos possíveis danos causados em um pavimento tais como: tráfego de veículos, efeitos ambientais, tempo de vida do pavimento e a condição atual do pavimento. A partir das relações causais estipuladas na figura 3, observa-se que as variáveis efeitos ambientais, danos associados ao tráfego e idade do pavimento apresentam relações causais positivas em relação a variável taxa de deterioração do pavimento. Assim, constata-se que todos esses efeitos contribuem para o aumento da degradação do pavimento.

Observa-se na figura 3 também que um "loop positivo" foi formado entre as variáveis condição do pavimento, efeito da condição atual do pavimento na taxa de deterioração e taxa de deterioração do pavimento. Este loop positivo reforça o desgaste causado pelas 
outras variáveis e nos permite afirmar que, na ausência de um loop negativo, o desgaste vai ocorrer de maneira exponencial até o total desgaste do pavimento.

A figura a seguir apresenta o diagrama causal para o modelo de deterioração do pavimento.

Figura 3 Diagrama Causal para a predição do desgaste do pavimento.

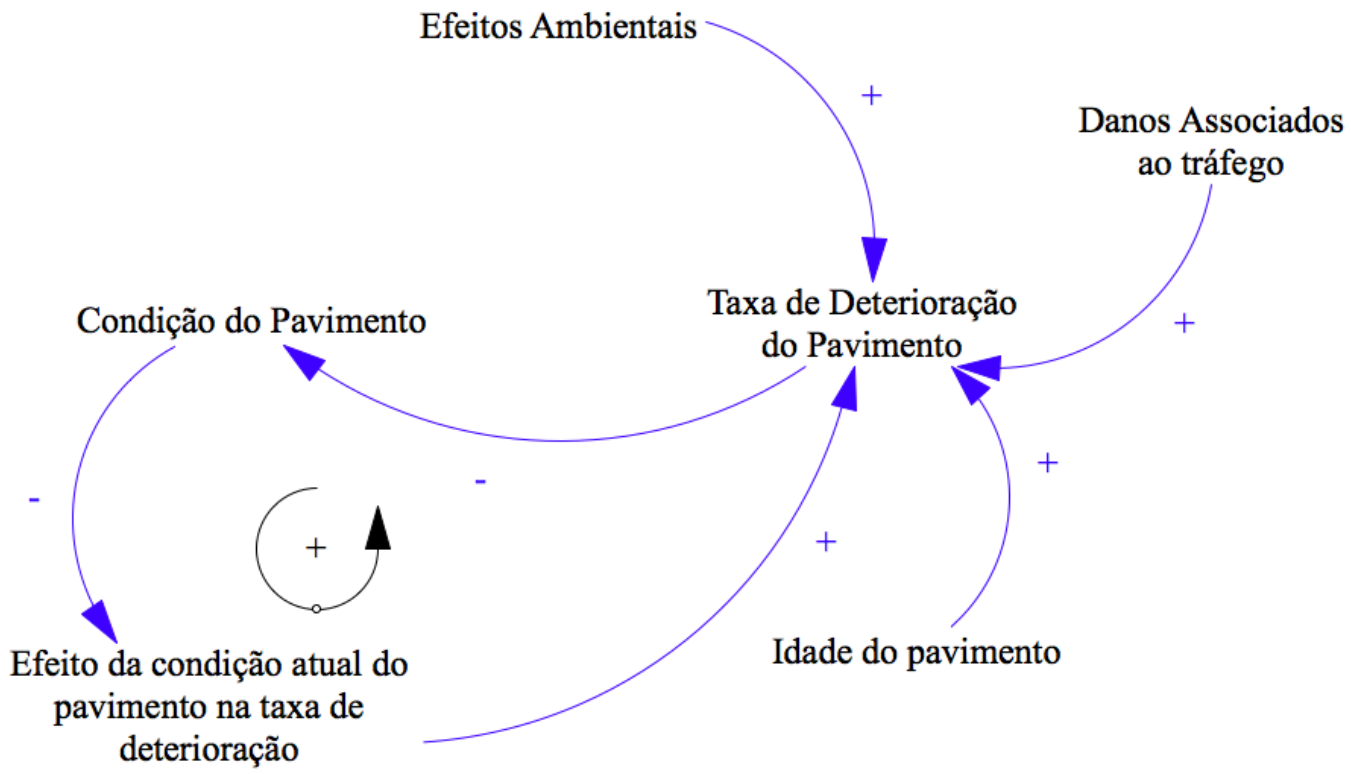

Fonte: Elaborado pelo autor

\subsection{DIAGRAMA DE FLUXO PARA A PREDIÇÃO DO DESGASTE DO PAVIMENTO}

A implementação dos diagramas causais oriundos da modelagem de problemas por meio da Dinâmica de Sistemas é feita por meio de softwares especializados. Neste trabalho o software utilizado foi o Vensim versão DSS.

Conforme apresentado na figura 4, a transcrição do modelo causal para o diagrama de fluxos é feita por meio de variáveis, fluxos e estoques.

O modelo proposto por Fallah Fini et al. (2015) foi adaptado para a realidade brasileira, portanto as formulações que envolvem as variáveis associadas aos danos no pavimento seguiram os padrões de dimensionamento de pavimento do Manual de Pavimentação do DNIT (2006) e serão explicadas em seguida.

\subsection{REPRESENTAÇÃO MATEMÁTICA DOS ESTOQUES E FLUXOS}

A integração ou acúmulo, cria o comportamento dinâmico dos sistemas. Na figura 4 temos representado como estoque a Qualidade do Pavimento e fluxo a taxa de deterioração. As outras variáveis, compõem a formulação da equação de fluxo. A equação 
do estoque pode ser representada por uma equação de diferenças finitas de primeira ordem, e pode ser expressa como;

$$
\text { Qualidade do pavimento }(t)=
$$

Qualidade do pavimento $\left(t_{0}\right)+\int_{t_{0}}^{t}($ fluxo de entrada $(t)-f l u x o$ de saida $(t)) \Delta t(1)$ em que to é o tempo de integração inicial e t é o tempo presente na integração. Para o problema proposto o modelo não contempla nenhum fluxo de entrada, o que é equivalente dizer que o modelo não contempla a recuperação do pavimento, apenas a deterioração (fluxo de saída). Adicionalmente, a equação (1) pode ser escrita em sua forma diferencial negligenciando o fluxo de entrada conforme mostrado a seguir.

$$
\frac{\text { dQualidade do pavimento }}{d t}=- \text { Taxa de deterioração }(t)
$$

A equação (2) é um problema de valor inicial e vários métodos estão disponíveis para resolver este problema numericamente. O software VENSIM permite a solução por meio dos métodos clássicos de Euler e Runge Kutta de quarta ordem.

Figura 4 Diagrama de fluxo para predição do desgaste do pavimento implementado no software Vensim DSS.

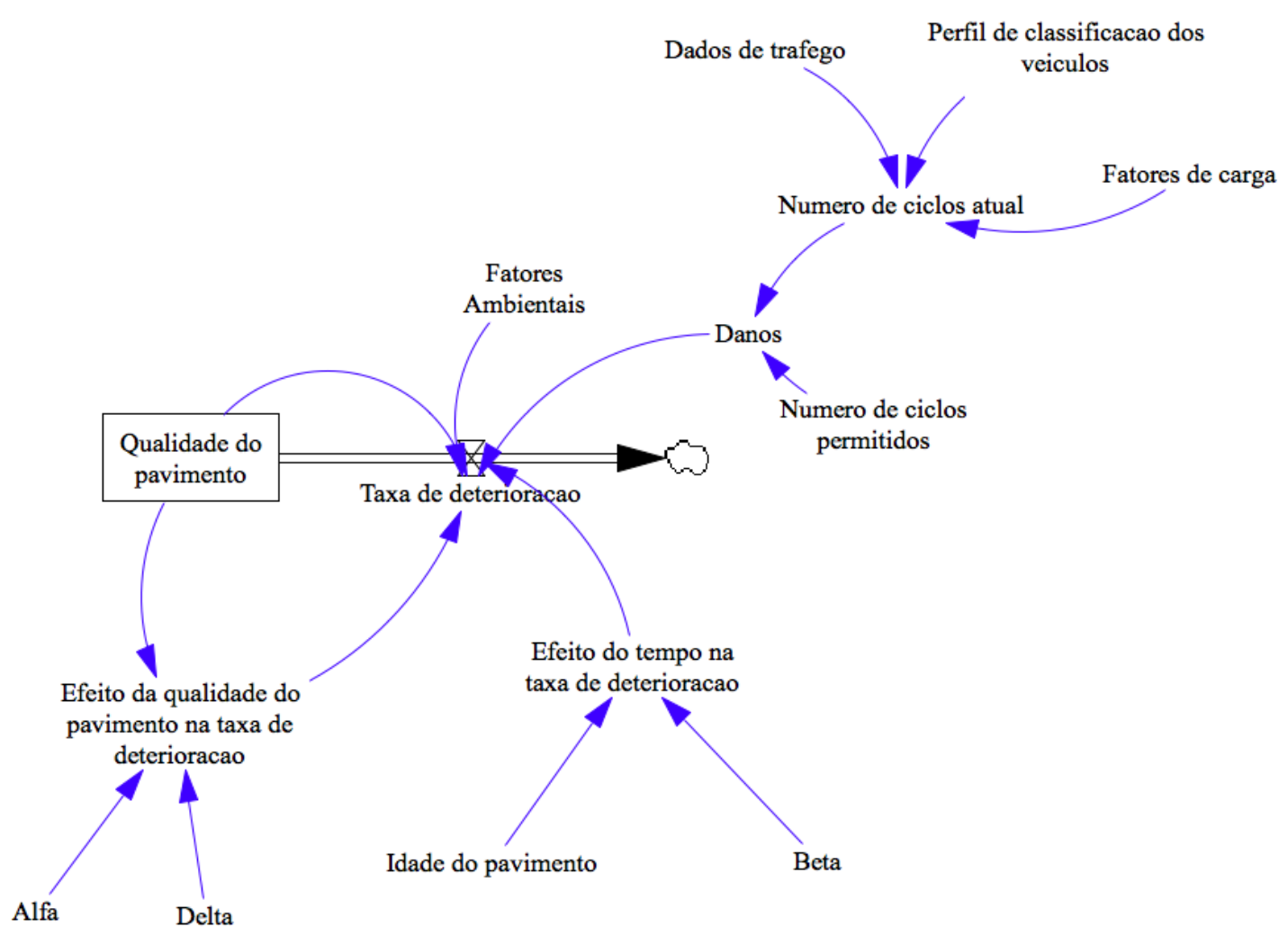

Fonte: Elaborado pelo autor 


\subsection{FORMULAÇÃO UTILIZADA NA IMPLEMENTAÇÃO DOS FLUXOS E VARIÁVEIS.}

Para construção do diagrama de fluxo para predição do desgaste do pavimento, a taxa de desgaste foi considerada em função de algumas variáveis que geraram equações vinculadas ao pavimento analisado DNIT (2006).

\subsubsection{Taxa de deterioração do pavimento}

Descreve a taxa de desgaste do pavimento em função das seguintes variáveis:

- Efeito da condição atual do pavimento na deterioração;

- Efeito do tempo na taxa de deterioração;

- Danos associados ao tráfego;

- Condição do pavimento.

- Fatores Ambientais

Portanto, a equação para a taxa de deterioração do pavimento pode ser expressa por meio do produto das cinco variáveis mencionadas nos marcadores acima.

\subsubsection{Efeito do tempo na taxa de deterioração}

O efeito do tempo de vida do pavimento na deterioração do mesmo é calculado por meio da equação (3) (FALLAH FINI et al. 2015):

$$
\begin{aligned}
& \text { Efeito do tempo na taxa de deterioração }= \\
& 1+(\text { idade do pavimento } / \text { vida útil do pavimento })^{\beta}
\end{aligned}
$$

em que a variável $\beta$ é ajustada aos dados experimentais de condição do pavimento em estudo. Tal metodologia de ajuste será descrita posteriormente.

\section{Efeito da condição atual do pavimento na deterioração}

A equação (4) procura representar o efeito da condição atual do pavimento no seu desgaste. Em outras palavras, a equação procura representar uma aceleração do desgaste do pavimento quando a condição do mesmo começa a cair. Este efeito é calculado por meio da equação (4) descrita a seguir:

Efeito da condição atual do pavimento na deterioração $=$

$1+\left(\alpha(1-\text { Qualidade do pavimento })^{\delta}\right)$ 


\subsubsection{Efeitos Ambientais}

Esta variável foi utilizada para representar efeitos ambientais diversos que colaboram com a degradação do pavimento e não são considerados de maneira explícita no modelo proposto. É um valor constante e será estimado através de dados experimentais.

2.5.4 Número permitido de ciclos, dados de tráfego, fator de carga e perfil de classificação dos veículos

O percentual de veículos de uma pesquisa com seus números de eixos correspondentes, quando multiplicados um pelo outro, nos dá o Fator de Eixo (FE) que transforma o tráfego desses veículos em número de passagens de eixos equivalentes conforme pode-se observar na expressão a seguir:

em que:

$$
F E=P_{2} \times 2+P_{3} \times 3+\ldots P_{n} \times n ;
$$

FE = fator de eixo;

$\mathrm{P}_{2}=\%$ de veículos de dois eixos;

$\mathrm{P}_{3}=\%$ de veículos de três eixos;

$P_{n}=\%$ de veículos de $n$ eixos;

Neste trabalho, o percentual de veículos de dois eixos foi da ordem de $90 \%$ e de veículos de três eixos da ordem de 10\%. Dessa forma, tem-se:

$$
\mathrm{FE}=0,9 \times 2+0,1 \times 3=2,1
$$

O Fator de Carga (FC) ou Fator de Equivalência de Operações (FEO) relaciona o efeito da passagem de qualquer tipo de veículo com o efeito de passagem de um veículo padrão. O cálculo do FC é feito por meio da equação (7):

$$
\mathrm{FC}=\Sigma \text { Equivalências } / 100
$$

Dando continuidade ao estudo proposto, deve-se observar que a utilização de ábacos do DNIT se faz necessária já que os mesmos determinam o Fator de Equivalência das operações em função da Carga por eixo em toneladas.

Conforme descrito no Manual de Pavimentos Asfálticos do DNIT, o VDM ou Volume Diário Anual de Tráfego (VMD) correspondente ao tráfego passado, presente e futuro da rodovia e a taxa de crescimento correspondente ao período da análise pode ser inferida pelo ajuste de uma equação (pelo método dos mínimos quadrados) aos dados existentes de modo a permitir não só o cálculo desse parâmetro ano a ano durante todo o período do projeto, como também em anos anteriores, desde a entrada em serviço do pavimento original.

Seguindo as metodologias padrões do DNIT(2006) os seguintes parâmetros foram assim calculados; 
Os seguintes parâmetros foram utilizados para calcular o valor das variáveis, dados de tráfego, fator de carga e perfil de classificação dos veículos.

Número permitido de ciclos $=3,2 \times 10^{7}$.

Dados de tráfego $(\mathrm{FE})=2,1$.

Fator de carga $(F C)=3,366$.

Perfil de classificação dos veículos $=7,0686$.

\subsubsection{Danos de tráfego}

A taxa de dano é calculada pela razão entre o número atual de ciclos e o número permitido de ciclos, em que o número atual de ciclos é calculado pelo produto das variáveis: Dados de Tráfego, Fator de carga e Perfil de Classificação dos veículos.

Índice de condição do Pavimento - ICP

O índice de condição do pavimento é calculado por:

ICP $=$ Qualidade do pavimento $\times 100$

\subsection{CALIBRAÇÃO DO MODELO}

A calibração do modelo é um processo de avaliação de parâmetros do modelo para obter uma correspondência entre o comportamento observado e o simulado. A calibração explicitamente é feita de tentativas de vincular um modelo matemático ao comportamento real, e é por isso que é desejável que seja feita com considerável rigor. A confiança em um determinado ser capaz de representar uma determinada realidade aumenta consideravelmente se o mesmo for capaz de gerar a dinâmica observada. Se o modelo não corresponder ao comportamento, então ele certamente será rejeitado.

Para o modelo proposto neste artigo, desejamos determinar parâmetros ideais, que, após uma busca em um intervalo de valores determinados, ofereçam o melhor ajuste de uma ou mais variáveis do modelo de dinâmica de sistemas para um conjunto de dados retirados do mundo real.

No presente modelo, os valores para as variáveis Alfa, Beta, Delta e Fatores Ambientais são incógnitas. O módulo de calibração presente no software VENSIM permite por meio de algoritmos de busca (“Search Techniques”) realizar a procura dos valores das varáveis incógnitas utilizando como entradas os intervalos permitidos para as variáveis e os dados experimentais para o modelo em questão. O problema de calibração/estimação de parâmetros se reduz então a um problema de minimização não linear com restrições. 
Os dados experimentais utilizados são referentes a de 8 segmentos/trechos de uma rodovia vicinal localizada no centro-oeste mineiro. Este trecho é referente a estrada MG 818, de responsabilidade do DER-MG, e liga os municípios de Florestal a Pará de MinasMG. Cada seguimento de trecho possui $800 \mathrm{~m}$, sendo que todos possuem a mesma característica técnico construtiva, contemplando uma seção transversal composta por subleito, reforço de subleito, sub-base, base e capa asfáltica em CBUQ (Concreto Betuminoso Usinado a Quente).

A tabela 1 a seguir mostra os dados experimentais de cada trecho utilizados para a calibração do modelo.

Tabela 1 Dados experimentais - Índice de Condição do Pavimento (\%) (Segmento 1 ao 6)

\begin{tabular}{ccccccc}
\hline Ano & Trecho 1 & Trecho 2 & Trecho 3 & Trecho 4 & Trecho 5 & Trecho 6 \\
\hline 2010 & 98 & 98 & 77 & 84 & 96 & 96 \\
2011 & 69 & 96 & 68 & 77 & 90 & 84 \\
2012 & 90 & 90 & 67 & 69 & 84 & 77 \\
2013 & 84 & 64 & 64 & 59 & 77 & 69 \\
2014 & 77 & 77 & 58 & 55 & 69 & 59 \\
2015 & 69 & 69 & 51 & 53 & 59 & 48 \\
\hline
\end{tabular}

\section{RESULTADOS}

\section{.1 CALIBRAÇÃO E VALIDAÇÃO DO MODELO DE DINÂMICA DE SISTEMAS}

Fallah Fini et al. (2015) sugerem que a estimativa dos parâmetros calculados pelo processo de calibração seja feita por meio de apenas um único valor para todos os trechos simulados. Entretanto, os parâmetros Alfa e Delta possuem relações causais diretas com a condição atual do pavimento. Portanto, utilizar o mesmo valor dos parâmetros para cada trecho seria equivalente a afirmar que os trechos representados pelo modelo se desgastem igualmente não considerando a condição individual de cada trecho.

A fim de considerar a condição individual de cada trecho da rodovia analisada, neste trabalho, foi feita a calibração dos parâmetros cada trecho encontrando-se, portanto, parâmetros distintos para cada um deles.

Pode-se observar na Tabela 2, os erros absolutos percentuais obtidos por meio do ajuste dos trechos, entre os anos de 2010 e 2015. 
DOI: 10.18605/2175-7275/cereus.v11n3p123-139
Teodoro, M. A.; Amantéa, R. P.; Santos, G. T; Vieira, S. L. A.; Rezende, R. D.

Um Modelo de Dinâmica de Sistemas para Previsão de Desgaste em Pavimentos Rodoviários no Brasil.

Tabela 2 Erro absoluto (\%) obtido pós calibração do modelo para os anos compreendidos entre 2010 e 2015.

\begin{tabular}{ccccccc}
\hline Ano & Trecho 1 & Trecho 2 & Trecho 3 & Trecho 4 & Trecho 5 & Trecho 6 \\
\hline 2010 & 0 & 0 & 0 & 0 & 0 & 0 \\
2011 & 0,2762 & 0,2762 & 0,2762 & 0,2762 & 0,2762 & 0,2762 \\
2012 & 3,479 & 3,479 & 3,479 & 3,479 & 3,479 & 3,479 \\
2013 & 4,406 & 4,406 & 4,406 & 4,406 & 4,406 & 4,406 \\
2014 & 2,526 & 2,526 & 2,526 & 2,526 & 2,526 & 2,526 \\
2015 & 3,855 & 3,855 & 3,855 & 3,855 & 3,855 & 3,855
\end{tabular}

O trecho 4 foi o trecho em que se constatou menor aderência aos dados experimentais. Tal fato se deve a oscilação dos dados experimentais obtidos nos anos de 2014 e 2015 em que o pavimento não mais se desgastou causando uma inflexão na dinâmica dos dados experimentais e consequente erro nos ajustes da curva simulada com a experimental. É importante citar que o trecho 4 foi o que apresentou maior erro, entretanto, em todos os anos o erro se maneteve inferior a 10\% para este trecho.

Para efeito de comparação e constatação da qualidade dos resultados obtidos, a figura 5 mostra os dados discretos experimentais e simulados para o trecho 4 e a figura 6 mostra os dados discretos experimentais e simulados para o trecho 6 . O trecho 6 foi considerado o trecho com melhor aderência aos dados experimentais.

Figura 5 Dados experimentais e simulados para o índice de qualidade do pavimento(\%) do trecho 4.

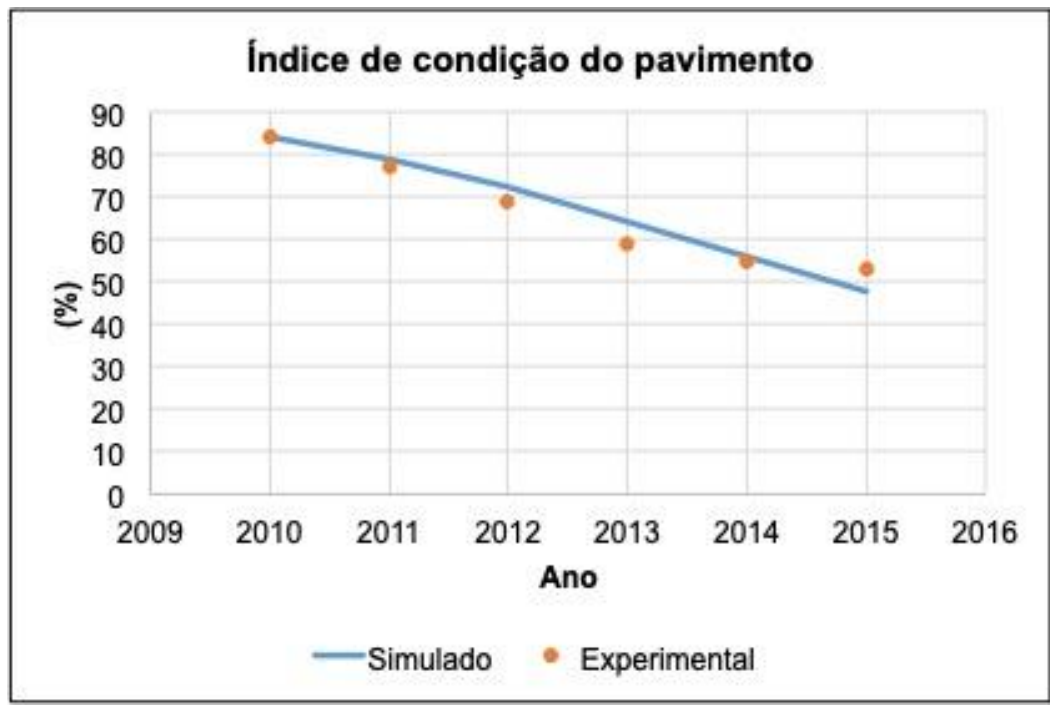

Fonte: Elaborado pelo autor. 
DOI: 10.18605/2175-7275/cereus.v11n3p123-139
Teodoro, M. A.; Amantéa, R. P.; Santos, G. T; Vieira, S. L. A.; Rezende, R. D.

Um Modelo de Dinâmica de Sistemas para Previsão de Desgaste em Pavimentos Rodoviários no Brasil.

Figura 6 Dados experimentais e simulados para o índice de qualidade do pavimento(\%) do trecho 6.

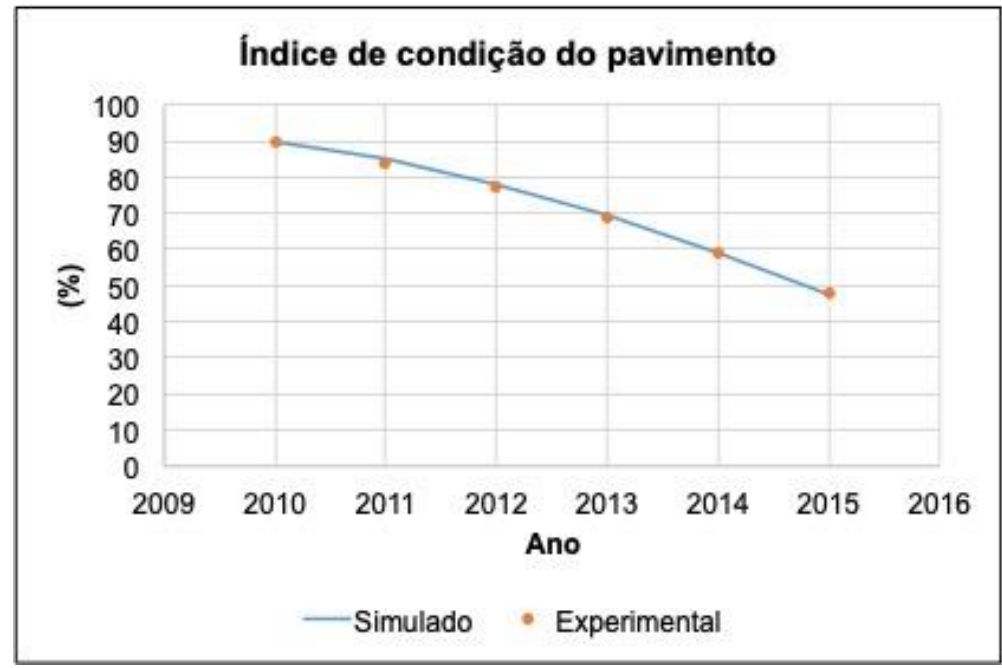

Fonte: Elaborado pelo autor.

\subsection{A DINÂMICA DO SISTEMA APÓS CALIBRAÇÃO}

A Figura 7, mostra a dinâmica do Índice de Condição do Pavimento (ICP) dos 6 trechos estudados. O conhecimento da dinâmica da degradação do pavimento possibilita um refinamento e orientação para os próximos passos referentes à gestão dos segmentos, como previsibilidade de intervenções preventivas, corretivas e restauração completa de trechos que atingiram ou irão atingir resultados inferiores a 50 no ICP, conforme limite apresentado na curva de degradação dos pavimentos asfálticos utilizados pelo DNIT.

Das seis curvas apresentadas na Figura 7, observa-se que os segmentos de pavimento representados pelas curvas de 1 e 5, um desgaste mais lento ao longo dos cinco anos analisados. Já os trechos 2, 3, 4 e 6 têm um comportamento mais acelerado de desgaste logo nos dois primeiros anos.

O estudo realizado neste trabalho abordou especificamente um trecho rodoviário construído em pavimento flexível que tem vida útil máxima estimada de 10 anos (com manutenção). Observa-se que o desenvolvimento das curvas do ICP demonstra uma degradação dentro da média de acordo com a literatura (manuais e procedimentos do DNIT) e expectativa esperada nos primeiros cinco anos. Diante do histórico do trecho, pode ser destacada a manutenção preventiva na via, evitando, assim, um aumento mais acentuado do desgaste da rodovia.

As curvas resultantes do modelo proposto e estudado podem auxiliar de forma significativa na tomada de decisões para direcionamento de equipes, recursos e priorização de segmentos pré-estabelecidos, proporcionando um incremento metodológico às práticas 
já existentes nos departamentos de fiscalização, controle e executivo de rodovias brasileiras.

Figura 7 - Dinâmica do ICP para os 6 trechos simulados.

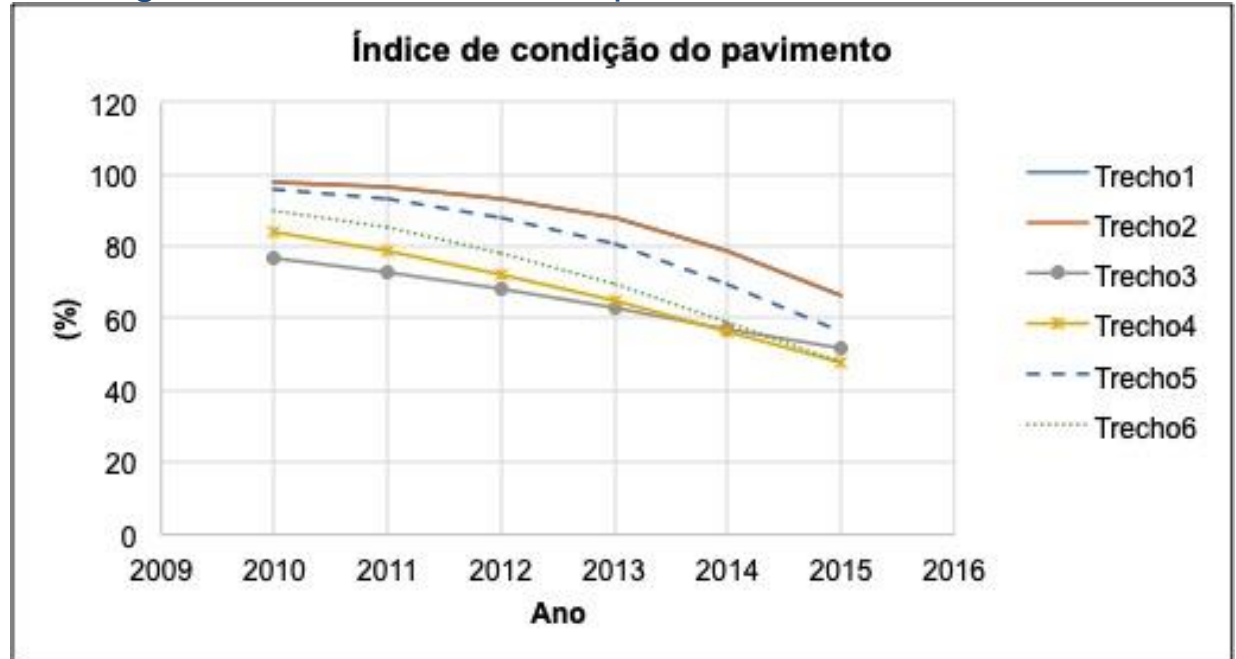

Fonte: Elaborado pelo autor

\section{CONSIDERAÇÓES FINAIS}

Neste trabalho, a modelagem de um Sistema de Desgaste de Pavimentos Rodoviários foi feita com o auxílio do software Vensim versão DSS, utilizando dados de 6 segmentos da rodovia MG 818, entre os municípios de Florestal e Pará de Minas-MG. Os resultados apresentados nas simulações dos modelos trabalhados foram satisfatórios e construtivos sob a ótica da engenharia rodoviária aliada à engenharia de sistemas.

Os desgastes e defeitos apresentados nas estruturas de pavimentos ocorrem com temporalidades e recorrências vinculadas a fatores que vão desde o mal dimensionamento de projeto, má execução da obra, chegando às variáveis de fatores de carga, clima, excesso de carga, desgaste natural dos materiais, entre outras.

Com a utilização de modelos de dinâmica de sistemas, foram realizados experimentos e simulações capazes de apresentar previsibilidades dentro de um espaço de tempo que apontam os momentos de possíveis ocorrências de desgastes no pavimento, podendo ser correlacionado à severidade e prováveis causas dos defeitos, viabilizando tomadas de decisões mitigadoras (manutenção preventiva, manutenção corretiva e restauração) de forma analítica e estratégica para cada aspecto apresentado. 
Do ponto de vista econômico financeiro, essa ferramenta pode ter no futuro grande impacto no que tange à otimização e previsibilidade de alocação de recursos, uma vez que a determinação das datas de cada tipo de ocorrência patológica na via propicia uma melhor programação de desembolso e empenho de recursos do caixa desses gerenciadores.

Os benefícios alcançados por esse modelo permitem um apoio substancial ao planejamento técnico, político e financeiro, provendo uma racional aplicação de recursos e mudando gradualmente a forma atual de gerência de pavimentos.

\section{REFERÉNCIAS}

BASTOS, J. B. C.; SOARES, J. B.; BARROSO, S. H. A. Considerações sobre a deformação permanente de pavimentos asfálticos no dimensionamento mecanísticoempírico a partir de ensaios acelerados e instrumentação em campo. In: Congresso de Ensino e Pesquisa em Transportes, 28, Curitiba, PR, 2014.

BOSURGI, G.; TRIFIRO, F. A Hybrid Approach for Pavement Maintenance Management of an Italian Motorway. Computing in Civil Engineering, 2005, p. 1-9.

CAREY JR., W.N.; IRICK, P.E. The pavement serviceability-performance concept. HRB Bulletin, n. 250, 1960 p. 40-58.

CAREY, W.; IRICK, P. The Pavement Serviceability - Performance Concept. Highway Research Board Special Report 61E, AASHO Road Test, Washington, D.C., 1962.

CONFEDERAÇÃO NACIONAL DOS TRANSPORTES. Pesquisa rodoviária 2018: relatório gerencial. Brasília, 2018. Disponível em: <http://www.cnt.org.br>. Acesso em: 03/06/19.

CRONEY, D. The design and performance of road pavement. London: Her Majesty's Stationery Office, 1977.

DEPARTAMENTO NACIONAL DE ESTRADAS DE RODAGEM. Manual de Pavimentação. Rio de Janeiro, 2006.

FALLAH FINI S.; TRIANTIS, K. Evaluating the productive effi ciency of highway maintenance operations: environmental and dynamic considerations. In XI European Workshop on Efficiency and Productivity Analysis, Pisa, Italy, 2009.

FALLAH FINI, S.; RAHMANDAD, H.; TRIANTIS K.; DE LA GARZA, J. M. Optimizing highway maintenance operations: Dynamic considerations. System Dynamics Review, July, 2010.

FALLAH FINI, S.; TRIANTIS, K.; DE LA GARZA. J. M.; SEAVER, W. Measuring the Efficiency of Highway Maintenance Contracting Strategies: A Bootstrapped NonParametric Meta-Frontier Approach. European Journal of Operational Research, v.219(1),2012, p.134-145. 
FALLAH FINI, S.; TRIANTIS, K.; RAHMANDAD, H.; DE LA GARZA, J. M.; Measuring Dynamic Efficiency of Highway Maintenance Operations. Omega, v.50,2015 p.18-28.

FRIEDMAN, Sheldon. The Effects of Dynamic Decision Making on Resource Allocation: The Case of Pavement Manegement. Dissertation in Partial Complementation of Ph.D. Program. Department of Social Science and Policy Studies and Department of Civil and Environmental Engineering. Worcester Polytechnic Institute. Worcester, Massachusetts. April 2003.

INSTITUTO BRASILEIRO DE GEOGRAFIA E ESTATÍSTICA. Mapa da logística dos transportes no Brasil.2014. Disponível em https://www.ibge.gov.br/geociencias/cartas-emapas/redes-geograficas/15793-logistica-dos-transportes.html?=\&t=downloads. Acesso em 03 de junho de 2019.

LEE, G.; YU, M.; WANG, L. DEA-Based Integrated Relationship of Returns to Scale An Application to Road Maintenance in Taiwan. Journal of Civil Engineering and Management. 18(5),2012, p.709-723.

LI, Z.; CHENG, C.; KWAN, M.; TONG, X.; TIAN, S. Identifying Asphalt Pavement Distress Using UAV LiDAR Point Cloud Data and Random Forest Classification. International Journal of Geo-Information. 8(39), 2019, p.1-26.

LOPES, Simone Becker. Gerência de Pavimentos Urbanos com Uso de Modelagem Dinâmica de Sistemas Diretamente Conectada com um SIG. Empresa Pública de Transporte e Circulação - EPTC - Porto Alegre - RS. Universidade de São Paulo - Escola de Engenharia de São Carlos, 2015.

MADEH PIRYONESI. S; EL-DIRABY, T. E. A Data Analytics Solution for Predicting the Condition of Roads Using the Most Affordable Attribute In: Canadian Society of Civil Engineering (CSCE), Vancouver, 2017.

SALINI, R.; INTELLI, P. Uma Abordagem Baseada em Inteligência Artificial para a Modelagem de Pavimentos Asfálticos. Manual de Técnicas de Pavimentação, vol. 1 e 2. Universidade do Minho - Escola de Engenharia - UT Austin. Portugal: Pini, 2010.

STERMAN J. Business Dynamics: Systems Thinking and Modeling for a Complex World. Irwin/ McGraw-Hill: Homewood, IL, 2000.

YANG, J.; JIANG, B. Experimental Study on Properties of Pervious Concrete Pavement Materials. Cement and Concrete Research, v. 33, 2003, p. 381-386. 